\title{
Strom aus Windkraft oder Stromausfall?
}

\section{Die Ergebnisse der Netz-Studie Deutschen Energie Agentur haben für einen öf- fentlichen Streit über die zukünftigen Kosten von Windenergie gesorgt. Sowohl Gegner als auch Befürworter der Windenergie sahen ihre Position durch die Er- gebnisse des Gutachtens gestärkt. Die folgende Analyse zeigt, wie solch ein scheinbarer Widerspruch möglich wird.}

S Von Roland Menges so genannte dena-Netzstudie. Diese Studie, die in der Langfassung einen Umfang von 500 Seiten und rund 700.000 Euro gekostet hat, wurde von einem Konsortium aus dem Energiewirtschaftlichen Institut der Universität Köln, dem Deutschen Windenergie-Institut in Wilhelmshaven und den Netzgesellschaften der großen Kraftwerksbetreiber (E.ON, RWE und Vattenfall) angefertigt. Betrachtet man das Auftraggeberkonsortium aus Verbänden und Unternehmen der Windkraftbranche, Netzbetreibern, Anlagenherstellern, Unternehmen der Kraftwerksbranche und dem Bundeswirtschaftsministerium, ist es nicht erstaunlich, dass es offenbar großen Streit hinter den Kulissen gab. So gelang es der dena nur durch die mehrmalige Verschiebung der Veröffentlichung und der Überarbeitung der Studie, zwischenzeitliche Meldungen zu dementieren, aus dem Gutachten könne die Aussage abgeleitet werden, der ,Ausbau der Windkraft macht Stromausfälle wahrscheinlicher" (FAZ vom 20.09.04), die postwendend vom Bundesverband WindEnergie als „,wissenschaftlicher Dilletantismus“ gebrandmarkt wurde. Auch die Umweltorganisation Greenpeace schaltete sich in die Diskussion ein und forderte prophylaktisch von den Energieversorgern, alle Versuche, aus dem Gutachten, „Stimmung gegen die Windenergie zu machen“ zu beenden.

\section{Ziel und zentrale Aussagen}

Die im September 2003 in Auftrag gegebene Studie hat das Ziel, eine ,verlässliche energiewirtschaftliche Planungsgrundlage“ für die Integration der Windenergie zu liefern und gleichzeitig eine Verständigung der beteiligten Akteure hier- über zu realisieren. Da offenbar während der Erstellung der Studie keine Einigung über die langfristige Integration der erneuerbaren Energien in das Energiesystem erzielt werden konnte, wurden diese langfristigen Überlegungen ausgeklammert und in eine Anschlussuntersuchung verlagert, so dass ,lediglich“ die Integration eines Ökostromanteils von 20 Prozent in das bestehende Verbundsystem betrachtet wird.

Die Studie leistet eine räumlich differenzierte Abbildung des mittelfristig prognostizierten Windenergieausbaus zu Land und zur See und die Zuordnung dieses Ausbaus zu den einzelnen Netzknoten. Im Vordergrund der Betrachtungen stehen somit

- die Auswirkungen der Windenergie auf die Übertragungsnetze (Studienteil „Netze“)

- und den übrigen Kraftwerkspark (Studienteil „Kraftwerke“).

Während beim landgestützten Ausbau der Windenergie bis zum Jahr 2015 mit einem Anstieg der Kapazitäten von 14,5 Gigawatt (GW) (in 2003) auf 26,2 GW gerechnet wird $(27,9 \mathrm{GW}$ in 2020) wird auf See im Jahr 2015 mit einer Kapazität von $9,8 \mathrm{GW}$ gerechnet $(20,4 \mathrm{GW}$ in 2020$)$. Um diese zusätzlichen Kapazitäten in das Verbundnetz zu integrieren, sind die Stromtrassen des Höchstspannungsnetzes auf einer Länge von $400 \mathrm{~km} \mathrm{zu}$ verstärken und auf einer Länge von $850 \mathrm{~km}$ neu zu bauen, was zu Kosten von rund 1,1 Milliarden führt. Ein umfassendes Systemmodell zum darüber hinausgehenden Ausbau der Offshore-Kapazitäten (nach 2015) soll jedoch erst in der Nachfolgestudie entwickelt werden. Hierbei soll die Errichtung einer hohen Anzahl paralleler ,Stichleitungen" verhindert werden. Dass dies tatsächlich jedoch auch schon für die Zeit vor 2015 ein wichtiges Ziel ist, zeigt die Tatsache, dass die derzeitig geplanten Offshore-Investitionsvorhaben über keine derart abgestimmten Netzplanungen verfügen, und vielmehr bereits jetzt einzelne Offshoreplaner (wie etwa die Gruppe ,Sandbank 24“) die Veröffentlichung von eigenen Netzstudien ankündigen, die die „Lüicke der dena-Studie“ schließen sollen - wenn auch wohl im Hinblick auf ihre jeweiligen spezifische Bedürfnisse.

Die Auswirkungen auf den Kraftwerkspark werden in der dena-Studie anhand von drei verschiedenen Szenarien abgebildet, die sich im Hinblick auf die angenommene langfristige Entwicklung der Energiepreise unterscheiden. Geht man beispielsweise von langfristig nicht-steigenden Energiepreisen aus und nimmt gleichzeitig bei Einführung des Kohlendioxid-Handels an, dass die Zertifikatpreise auf 12,5 Euro je Tonne im Jahr 2015 steigen werden, so wird windenergiebedingt insbesondere die Stromerzeugung aus Stein- und Braunkohlekraftwerken abnehmen. Geht man hingegen c.p. von einem stärkeren Anstieg des Öl- und Gaspreises aus, so findet die Verschiebung eher zu Lasten der Erdgaskraftwerke statt. Grundsätzlich gehen die Gutachter davon aus, dass für den hier betrachteten Windenergieausbau keine zusätzlichen Kraftwerke zur Bereitsellung der Regel- und Reserveleistung zu installieren sind. $\mathrm{Zu}$ ähnlichen Ergebnissen kommt beispielsweise eine jüngere Untersuchung des Fraunhofer Instituts, die zeigt, dass - ausgehend von den derzeitigen Strukturen - die windenergiebedingten Substitutionseffekte im Mittellastbereich von Steinkohle-, Braunkohle- und Ergaskraftwerken zu verzeichnen sind, und dass die „technischen Möglichkeiten zur Integration auch großer Mengen fluktuierender Erzeugung vorhanden sind" (1).

Die Mehrkosten für die ab dem Jahr 2003 eingespeisten Windstrommengen belaufen sich - je nach angenommenem Energiepreisszenario - im Jahr 2015 auf 1,6 bis 2,3 Milliarden Euro. Die Kohlendioxid-Vermeidungskosten sinken von anfänglich deutlich höheren Beträgen bis zum Jahr 2015 auf einen Wert zwischen 41 und 77 Euro je Tonne. Nach den Aussagen der Studie werden durch den Ausbau der Windkraft im Jahr 2015 zwischen 20 und 40 Millionen Tonnen Kohlendioxid-Emissionen vermieden.

\section{Diskussion des Gutachtens}

Erwartungsgemäß interpretieren alle Kommentatoren die Ergebnisse des Gutachtens vor dem Hintergrund ihrer jeweiligen Interessen. Während Bundesumweltminister Trittin ähnlich wie der Bundesverband WindEnergie betont, der Ausbau 
der Windenergie lasse sich bei moderatem Ausbau der Netze kostengünstig in das deutsche Stromnetz integrieren, ziehen beispielsweise für den umweltpolitischen Sprecher CDU/CSUBundestagsfraktion dieselben Investitionen in Netzstabilität und Versorgungssicherheit ,hohe Investitionskosten“ nach sich. Die FDP-Bundestagsfraktion sieht die im Gutachten genannten Kohlendioxid-Vermeidungskosten als unverhältnimäßig hoch an - insbesondere in Relation zum für das Jahr 2015 angenommenen Zertifikatpreis von 12,5 Euro. Dies sind allerdings Bewertungsfragen, die sich bekanntlich einer wissenschaftlichen Grundlage entziehen.

Problematischer sind hingegen Kritikpunkte, die sich auf methodische Aspekte der Studie beziehen. So wird von den verschiedenen Verbänden der erneuerbaren Energien kritisiert, dass nur in einem der drei Szenarien eine moderate Erhöhung der Energiepreise angenommen wird. Klar ist, dass bei stärker steigenden Energiepreisen, wie sie beispielsweise auch von der Deutschen Bank angenommen werden, die gesellschaftlichen Kosten des Windenergieausbaus entsprechend geringer sind als im dena-Gutachten (2). Ob jedoch die in diesem Zusammenhang häufig angenommene Kombination eines langfristig stetig steigenden Preises der fossilen Energieträger mit einem stetig sinkenden Preispfad der erneuerbaren Energien (Degressivität der Tarife, Kostendegression bei den Anlagen), die zu einer Subventionierung der fossilen Energien führt, sobald sich beide Preispfade schneiden, tatsächlich eine reale Perspektive oder eher einen wünschenswerten Zustand beschreibt, kann ebenfalls nicht mit Sicherheit beantwortet werden.

\section{Off-Shore Anlagen}

Darüber hinaus kritisiert der Bundesverband WindEnergie, dass für die Offshore-Anlagen auch nach 2010 eine erhöhte Vergütung angenommen wird, während eher mit einer Degression der Tarife und somit geringeren Kosten des Ausbaus der Windenergie zu rechnen sei. Tatsächlich scheinen beim Bau der Offshore-Anlagen und bei deren Netzanschluss die größten Unsicherheiten der dena-Studie zu liegen, da es hier allenfalls Planungen aber noch keine realen Erfahrungen gibt. Eine Studie von Jeske und Hirschhausen kommt beispielsweise im Fall der Simulation von drei repräsentativen Offshore-Windparks in Nord- und Ostsee zu dem Ergebnis, dass die derzeitigen Einspeisungsvergütungen des EEGs nicht hoch genug sind, um einen betriebswirtschaftlich rentablen
Betrieb der Anlagen zu ermöglichen (3). Da die Wahrscheinlichkeit zukünftiger Kostendegressionen nicht ausreiche, die kurzfristig anstehenden Investitionsentscheidungen nachhaltig zu beeinflussen, müssten weitere politische Maßnahmen ergriffen werden. Auch diverse andere Publikationen gehen davon aus, dass die betriebswirtschaftliche Frage, welche Erträge ein OffshoreWindpark innerhalb einer Lebensdauer von 20 oder 25 Jahren erzielen könne, bei weitem noch nicht gelöst sind. Dies ist kein Argument gegen den politisch erwünschten Ausbau der OffshoreWindkraft. Grundsätzlich ist für diese Investitionen in Offshore-Windparks und die dazugehörigen Netzanbindungen und -verstärkungen jedoch privates Kapital zu moblisieren. Dieses ist letztlich ausschließlich an der Rentabilität und einer angemessenen Kapitalverzinsung orientiert und kann nicht - wie noch in Monopolzeiten - per Regulierung erzwungen werden. Neben der Regulierung der Einspeisungstarife kommt somit auch der zukünftigen Regulierung der Netznutzungsentgelte wie zum Beispiel Nettosubstanzerhaltung versus Realkapitalerhaltung eine wichtige Rolle zu. Aus der Perspektive der von den dena-Gutachtern angestrebten ,langfristigen energiewirtschaftlichen Planungen" dürften die vom EEG und EnWG bestimmten Größen jedoch eher endogener als exogener Natur sein. Wenn hingegen die dena-Studie beispielsweise davon ausgeht, dass die Investitionskosten für die Seekabelanschlüsse, die bis zum Jahr 2015 fünf Milliarden Euro betragen und bis zum Jahr 2020 auf elf bis 12 Milliarden Euro ansteigen werden, schon von den (derzeit geltenden) EEG-Tarifen gedeckt seien, so zeigt dies, welche kostenmäßigen Unsicherheiten sich hinter diesen Kalkulationen noch verbergen. Manche Kommentatoren und auch die dena selbst weisen darauf hin, dass ein für die Integration der Windenergie zu erweiterndes Verbundnetz auch für den internationalen Stromhandel zur Verfü-

\section{Inserieren Sie in Ökologisches Wirtschaften!}

Sprechen Sie genau Ihre Zielgruppe an. Erscheint 5 x jährlich.

Fordern Sie noch heute die Mediadaten an!

Frau Dagmar Huber berät Sie gerne.

\section{Kontakt: \\ Dagmar Huber}

Waltherstraße 29, 80337 München

Fon 089/544 184 12, Fax 089/544 18449

E-Mail: huber@oekom.de gung steht. Dies deckt sich mit den Aussagen von Energieunternehmen, dass derzeit beispielsweise erhebliche Teile der Übertragungskapazitäten zwischen Deutschland und den Niederlanden wegen der Einspeisung von Onshore-Windenergie nicht genutzt werden können und insofern den Stromhandel begrenzen. Dieses Argument richtet sich jedoch nicht auf die Höhe der Kosten des Netzausbaus, sondern liefert lediglich ein zusätzliches Argument für die volkswirtschaftliche Notwendigkeit und Vorteilhaftigkeit der Investitionen.

Bei der Gesamtbewertung des Gutachtens erscheint es wichtig, eine klare Trennlinie zwischen wissenschaftlichen Aussagen und den zu treffenden politischen Wertungen und Entscheidungen zu ziehen. Es wäre illusorisch, von dem vorgelegten Gutachten mehr als wissenschaftlich überprüfbare Aussagen über die technische Machbarkeit des Netzausbaus und einen zu erwartenden Kostenrahmen zu erwarten und dieses etwa als politisches Konsenspapier zu betrachten. Wissenschaftliche Studien stellen keine deterministischen Aussagen über zukünftige Entwicklungen dar, sondern können allenfalls als Entscheidungshilfen dienen. Schon Brennan und Buchanan haben gezeigt, dass die Vorstellung, politische Entscheidungen über das Gemeinwohl könnten wie wissenschaftliche Aussagen aus einem überprüfbaren Modell abgeleitet werden, letztlich undemokratisch ist (4).

\section{Anmerkungen}

(1) Klobasa, M./ Ragwitz, M: Simulation der Windeinspeisung in Deutschland und Rückwirkungen auf den Kraftwerksbetrieb. Beitrag zur IEWT 2005, Wien 2005.

(2) Deutsche Bank Research ( $H g$.$) : Energieperspektiven$ nach dem Ölzeitalter. Studie Nr. 309, Frankfurt a. M. 2004 (3) Jeske, T./ von Hirschhausen, C.: Offshore Windenergie - Studie zur betriebswirtschaftlichen Rentabilität von Offshore-Windparks in der Deutschen Nord- und Ostsee. Beitrag zur 4. Internationalen Energiewirtschaftstagung IEWT 2005, 16.-18. Februar 2005, Wien 2005.

(4) Brennan, G./ Buchanan, J. M.: Die Begründung von Regeln - Konstitutionelle Politische Ökonomie. Tübingen 1993.

\section{Der Autor}

Dr. Roland Menges ist wissenschaftlicher Mitarbeiter im Bereich Energie und Umweltmanagement an der Universität Flensburg.

Kontakt: E-Mail: r.menges@essh.de 
(c) 20I0 Authors; licensee IÖW and oekom verlag. This is an article distributed under the terms of the Creative Commons Attribution Non-Commercial No Derivates License (http://creativecommons.org/licenses/by-nc-nd/3.o/), which permits unrestricted use, distribution, and reproduction in any medium, provided the original work is properly cited. 